\title{
The Treatment of Preschool Mood Disorders
}

\section{Melissa DeFilippis, MD* \\ Karen Dineen Wagner, MD, PhD}

\author{
Address \\ *Department of Child and Adolescent Psychiatry, The University of Texas Medical \\ Branch, 301 University Blvd, Galveston, TX 77555-0188, USA \\ Email: msdefili@utmb.edu
}

Published online: 29 January 2015

(C) Springer International Publishing AG 2015

This article is part of the Topical Collection on Child and Adolescent Psychiatry

Keywords Preschool - Bipolar disorder - Depression - Mood disorders - Antidepressants - Atypical antipsychotics . Psychoeducation - Parent-child interaction therapy

\section{Opinion statement}

Pediatric mood disorders are a genuine concern within the clinical setting, and they cause significant morbidity among children and adolescents. Evidence supports the presence of mood disorders in even very young, preschool-aged children, but they can be difficult to diagnose accurately in this population. They may present somewhat differently, especially with regards to duration and timing of mood symptoms. For example, clinically significant depression may be missed in this age group when using the strict 2-week duration criterion, and clinicians will identify these significant cases more successfully when using a proposed 1-week duration criterion. Mood disorders in the preschool population may also present with more non-specific symptoms (i.e., irritability), and developmental aspects encountered in this age group may also contribute to diagnostic uncertainty. There is considerable controversy surrounding the diagnosis of mood disorders, especially bipolar disorder, in young children. Children seem to present more often with fairly rapid cycling between mood states, and this seems especially true with preschool-aged children. This can lead to misdiagnosis and incorrect treatment decisions. Evidence examining these diagnostic issues is limited, but it supports the validity of mood disorder diagnoses in this young population. Evidence regarding treatment in this age group is even more limited. Treatment of mood disorders in the preschool age should begin with psychosocial interventions, first. These include parent-training, psychoeducation, and cognitivebehavioral therapy. Medication studies in this age group are lacking, and those available are largely open label trials or case series. When medication is necessary, special attention to dosage should be made, with starting doses cut in half from regular pediatric dosing recommendations to avoid adverse effects. Fluoxetine should be considered as first-line pharmacotherapy in preschool children with depression, due to the available safety and efficacy data in school-aged children. Atypical antipsychotics have the most evidence supporting their use in the treatment of bipolar disorder in preschool-aged children and should considered first, in cases where pharmacotherapy is necessary. While there is a 
definite need for additional research in both the diagnosis and treatment of preschool mood disorders, clinicians should use what available evidence there is to aide them in treating this very young population.

\section{Introduction}

\section{Prevalence of Mood Disorders}

It is well-established that mood disorders are prevalent among school-aged children and adolescents, but few studies have examined the prevalence or management of mood disorders in preschool-aged children. The available studies support the validity of these diagnoses in this age group. They also highlight the need for more research examining the diagnosis and treatment of mood disorders in this population. A population-based study examining risk factors for depression in preschool-aged children showed a prevalence of depressive symptoms in $6 \%$ of children aged 5-7 years $(n=653)$ [1]. Factors associated with increased risk of depressive symptoms included parental separation, comorbid behavioral problems, and developmental motor and speech disorders. A study that included 796 four-year-old children sampled from both public schools and primary care clinic settings showed a prevalence of $<1 \%$ for either major depressive disorder or dysthymia [2•]. Epidemiological evidence specifically examining bipolar disorder in the preschool population is limited. A case series including 40 children under the age of 5 who consecutively presented to a mental health clinic for any reason found 11 children (27.5\%) met criteria for mania [3]. Luby and Belden examined a communitybased sample of 303 preschoolers (aged 3-6 years) and found $26(8.6 \%)$ met criteria for bipolar disorder [4]. Higher impairment was associated with a diagnosis of bipolar disorder when compared to the other diagnostic groups and healthy controls.

\section{Predictive Factors}

Associated risk factors for depression include lower socioeconomic status, stress and family conflict, caregiver depression, parenting, and temperament (negative affect and poor sensory regulation) [5]. Irritability is a common symptom among children and may be associated with the development of mood disorders. In a longitudinal study of 462 three-year-old children, significant irritability was determined using the Preschool Age Psychiatric Assessment (PAPA) [6, 7]. Irritability at age 3 predicted depression and oppositional defiant disorder (ODD) symptoms at age 6 as well as functional impairment. Baseline irritability was also associated with a family history of anxiety and depression. Certain temperament factors seem to be related to depression in preschool-aged children. A study of 541 three-year-olds showed temperament factors of dysphoria and low exuberance levels to be associated with depressive disorders (including major depressive disorder, dysthymia, and depressive disorder not otherwise specified) [8]. A study examining the continuity of preschool depression included 246 children whose parents were initially interviewed using the PAPA when the children were between the ages of 3 years and 7 years $[9 \bullet \bullet]$. Parents were interviewed again later, at age 8 years or older along with the children, who were interviewed using the Child and Adolescent Psychiatric Assessment (CAPA) [10, 11]. Children diagnosed with preschool-onset depression were 2.5 times more likely to be diagnosed with school-age depression when compared to preschool children without depression. This suggests diagnostic continuity of depression from preschool age to school age.

\section{Diagnosis of Depression in Preschoolers}

Despite limited research in this very young population, emerging evidence supports the validity of major depressive disorder in preschool-aged children. Symptoms which seem highly specific for depression in this age group are guilt and extreme fatigue, and the symptoms cause functional impairment [12]. Evidence suggests that DSM-IV criteria can be used to correctly diagnose major depression in preschool children; however, a significant portion of children with clinically significant symptoms and functional impairment may be missed without using some developmental modifications to the criteria [13]. Many of the modifications are slight, and focus on play themes (i.e., self-destructive or excessive guilt themes evident in play), but still require five or more symptoms for a diagnosis. Another focus has been on the duration criteria for major depression. When the 2-week duration criteria are applied to preschool children, a significant number of clinically affected children are missed [14]. In a 2-year longitudinal study, 74 depressed preschool children and 77 healthy preschool children were followed up to see who developed a diagnosis of major depressive disorder (MDD) at follow-up 
[15]. The group of depressed preschool children included those meeting full DSM-IV criteria for MDD and those who met all the criteria except the 2-week duration criterion. The study found no difference in severity of depression at follow-up between these two groups, suggesting children failing to meet the 2-week duration criterion are at similar risk for later clinically significant depression as those who met this criterion in the preschool years. This supports the proposal that modifications to the duration criterion be used for preschool-aged children with depression so as not to miss clinically relevant symptoms in this vulnerable population.

\section{Diagnosis of Bipolar Disorder in Preschoolers}

Evidence supports the presence of bipolar disorder in preschool-aged children using DSM-IV criteria, and symptoms of hypersexuality, grandiosity, and elation seem to be specific markers for bipolar disorder in this population [4]. A study exploring possible diagnostic differences by age in youths diagnosed with bipolar disorder included a preschool-aged group (aged 47 years) [16]. The study showed very little age effects on manic symptoms. The only significant difference in manic symptoms was a slight decrease in motor activity and aggressive behavior with age, as measured by the Young Mania Rating Scale (YMRS) and K-SADS Mania Rating
Scale (KMRS) $[17,18]$. Depressive symptoms gradually increased with age. The findings suggest the core symptoms of bipolar disorder can be used to reliably diagnose mania in very young children as well as adolescents. A study examining the differences between unipolar and bipolar depression in preschool children found more severe depressive symptoms in the children with bipolar depression when compared to those with unipolar depression [19]. The study also showed a higher comorbidity of ADHD, ODD, conduct disorder, and anxiety disorders among the children with bipolar depression when compared to the children with unipolar depression.

Proposals have been made to adjust the definition of bipolar I disorder in the pediatric population, especially in the preschool population. This is based on evidence that shows young children are more likely to have brief periods of mania which occur multiple times a day, also called ultradian cycling, and suggests the need for duration criteria modification for pediatric bipolar disorder [20]. This group also suggests the definitions for cycle and episode be defined separately for the pediatric population, instead of being used interchangeably as in adult bipolar disorder [21]. They suggest episodes should be defined as the duration of either one mood state or of cycling of mood states and cycles be defined by mood switches occurring daily or every few days within an episode.

\section{Treatment}

In preschool-aged children with mood disorders, psychosocial treatments have the most evidence supporting their use; however, there is little evidence specific to the preschool population, with many studies only including school-aged children and older. Treatments studied include psychosocial interventions, psychoeducation, and cognitive-behavioral treatments. Pharmacologic treatments have not been sufficiently studied in preschool-aged children, and many studies including this age group have also included school-aged children and adolescents. There is also a lack of randomized controlled trials in this age group, with most studies being open-label trials or case series.

\section{Major Depressive Disorder}

\section{Psychosocial Interventions}


conducted supporting the use of Parent-Child Interaction Therapy Emotion Development (PCIT-ED) in this patient population. PCIT-ED is adapted from PCIT, which focuses on improving the parent-child relationship as well as teaching parents how to set effective limits in children with disruptive behaviors [22]. PCIT-ED is focused on the parent and child relationship using in vivo training of the parent [23]. It also targets emotional identification and regulation. The goal is to encourage positive emotions and affects and to reduce negative emotions through coaching of the parents. The therapy includes 14 sessions along with homework assignments.

In an open pilot study of eight preschool-aged children (aged 3-5 years), PCIT-ED was shown to be effective in reducing severity of MDD symptoms, as determined by scores on the PAPA, with an effect size of 1.28 [24]. Out of the seven preschoolers who completed all 14 sessions, five no longer met criteria for MDD post-treatment.

A pilot randomized controlled study compared the efficacy of PCIT-ED to that of psychoeducation in the treatment of major depressive disorder in

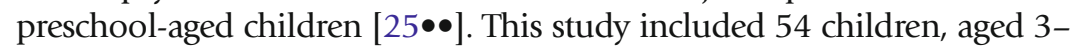
7 years, who met criteria for major depressive disorder, with 29 participants completing the study. Primary outcome measures included depression severity scores on both the PAPA and the Preschool Feelings Checklist-Scale Version (PFC-S) [26]. Both treatment groups showed a significant decline in depression severity as measured by the PFC-S, but the two groups did not differ significantly from each other. When using the PAPA, only the PCIT-ED group showed significant declines in MDD severity, though there was no significant difference in severity scores between the two groups. Secondary outcome measures showed significant improvements in emotional development and executive functioning in the PCIT-ED group when compared to the psychoeducation group. The completion rate was significantly higher in the PCIT-ED group than the psychoeducation group. Despite the limited power of this study due to the small size, it suggests PCIT-ED as a feasible and effective treatment for preschool-aged children with depression.

PCIT requires the involvement and training of parents or caregivers. Core modules of PCIT, including Child-Directed Interaction (CDI) and ParentDirected Interaction (PDI), are included but limited to four sessions. The Emotional Development (ED) module is specific to PCIT-ED and consists of six sessions. It focuses on emotional competence and regulation, with the parents serving as guides for the children. In vivo coaching by the therapist occurs throughout the sessions.

Treatment Duration

Treatment consists of three modules, over 14 sessions in 12 weeks.

PCIT-ED requires direct involvement of parents/caregivers, and would be contraindicated when this involvement cannot be assured. 


\section{Cognitive-Behavioral Therapy}

Cognitive-behavioral therapy has been studied more extensively in older children and adolescents with depression, and there have been a few studies in younger school-aged children. It has not yet been studied in the preschool population, and it is not clear what modifications may be needed to effectively address depression in this very young age group. A study examined the efficacy of cognitive-behavioral treatment (CBT) in a group of 57 youths, aged 8-15 years, with depressive disorders [27]. The participants were randomized to receive either usual care (UC) or a manual-based CBT program. Though there was no difference between the two groups in symptoms severity at endpoint (with $75 \%$ of youths no longer meeting criteria for a depressive disorder), the CBT group reached this outcome sooner, had higher parent-rated therapeutic alliance, was less likely to require additional services (including medication), and was less costly. Symptom severity was measured using the Children's Depression Inventory (CDI) and Child Behavior Checklist (CBCL) $[28,29]$. An earlier study by this same group examined the efficacy of CBT versus a control treatment (no treatment) in 48 children in grades 3-6, with mild to moderate depression [30]. The treatment group showed significantly greater reductions than the control group in severity of symptoms as measured by the CDI and Children Depression Rating Scale-Revised Version (CDRSR) at endpoint and at 9-month follow-up.

Special Points

Cognitive-behavioral therapy has been studied in older children and adolescents with depression, with very few studies including younger school-aged children. The few studies including children under the age of 10 have shown this as a promising treatment for depression; however, findings cannot be generalized to the preschool population.

Treatment Duration

Duration of treatment varies, but it typically involves 12 or more sessions.

Contraindications

There are no contraindications.

Pharmacological Interventions

\section{Selective Serotonin Reuptake Inhibitors-Fluoxetine}

There are currently no medications approved for the treatment of depression in preschool-aged children. The American Academy of Child and Adolescent Psychiatry (AACAP) has developed treatment guidelines for preschool-aged children $[31 \bullet \bullet]$. These include first obtaining a comprehensive, multiinformant assessment, then proceeding with evidence-based psychotherapy treatments. Medications are only recommended for children who still experience moderate to severe symptoms and significant functional impairment after psychotherapy, and only in cases when there is a reasonable expectation that 


\section{Contraindications}

psychiatric medications may be beneficial. The guidelines also emphasize the importance of close monitoring of treatment effects. They recommend discontinuation of medication(s) after a period of successful treatment to assess need for further treatment.

If medication is necessary due to symptom severity and functional impairment, fluoxetine would be the initial treatment recommendation for depression in this patient population, based on efficacy in school-aged children 8 years and older. A meta-analysis of randomized controlled studies of SSRI use in children and adolescents aged 5 to 18 years showed fluoxetine as the only SSRI with a favorable efficacy-to-safety ratio among children [32]. A more recent metaanalysis also showed fluoxetine to be the only SSRI to be effective in the treatment of depression among children and highlighted the increased risk of suicidal ideation among children and adolescents treated with antidepressants [33].

SSRIs should not be the initial treatment of choice in preschool children with bipolar disorder, due to the risk of antidepressant-induced manic episodes [34]. SSRIs are contraindicated in individuals with documented hypersensitivity to the drugs.

Drug Interactions

Fluoxetine is metabolized by the P450 2D6 enzyme system, and potential interactions exist with other drugs metabolized by this system, including tricyclics and other SSRIs. Other possible drug interactions include tryptophan, diazepam, carbamazepine, and phenytoin [35].

Side Effects

Common side effects of SSRIs include gastrointestinal complaints (nausea, abdominal pain), headache, insomnia, somnolence, and dry mouth [36-39].

Special Points

Fluoxetine is the only medication approved for use in children $<12$ years of age with major depression. In children $<8$ years of age, psychotherapy should be considered before medication. In preschool-aged children with depression, fluoxetine should be dosed at half the recommended dose for children (initial dose not exceeding $5 \mathrm{mg}$ ). Fluoxetine is available in oral solution form, $20 \mathrm{mg} / 5 \mathrm{~mL}$.

Cost

Fluoxetine is available in generic form and is inexpensive.

Bipolar Disorder

\section{Psychosocial Interventions}

Psychosocial therapies should be considered first-line treatment for preschoolaged children with bipolar disorder when possible, due to safety concerns with 
pharmacological treatments in this very young age group. However, many of the psychosocial interventions developed for use in bipolar disorder are meant to be used along with medication. Psychosocial interventions have not been studied much in young children with bipolar disorder, and most interventions have been focused on adolescents. There are a few treatments that seem to be promising in school-aged children.

\section{Child and Family-Focused Cognitive-Behavioral Treatment}

Child and family-focused cognitive-behavioral treatment (CFF-CBT), also called the RAINBOW Program, has been studied in children with bipolar disorder as young as 5 years old [40•]. The studies thus far have been open, feasibility studies but have shown promising results. The focus of CFF-CBT is on psychoeducation, lowering expressed emotion in caregivers, crisis management, and relapse prevention, while taking into consideration developmental factors present in pediatric bipolar disorder, including family and school stressors. The mnemonic, RAINBOW, is used to help children and families to remember the skills taught in this therapy. They stand for routine, affect regulation, "I can do it" (positive selftalk), no negative thoughts/live in the now, be a good friend/balanced lifestyle for parents, "oh, how can we solve the problem" (problem-solving skills), and ways to get support. These interventions are presented over 12 sessions.

In an open-trial, feasibility study of CFF-CBT, 34 patients with bipolar disorder, aged 5-17 years, showed significant reductions in symptom severity, as measured by the Clinical Global Impression Scales for Bipolar Disorder (CGI-BP) $[40 \bullet, 41]$. An open pilot study of a maintenance model of this same intervention was conducted with the same 34 participants and their families [42•]. It included medication management and booster sessions which ranged from once per week to once every 3 months over the course of 3 years. The results showed continued maintenance of symptom improvement over the course of the 3 years of treatment and suggested maintenance CFF-CBT as a feasible treatment option for children with bipolar disorder. A pilot study examining the efficacy of CFF-CBT for pediatric bipolar disorder in a group format has also shown promising results [43]. A randomized controlled trial examining the efficacy of CFF-CBT for the treatment of bipolar disorder in 69 children aged 7-13 years showed it to be more effective in reducing parentreported mania symptoms than psychotherapy as usual [44].

CFF-CBT is provided as both an acute treatment and a maintenance treatment for children with bipolar disorder. Booster sessions focus on reviewing the core components of CFF-CBT with the children and parents. CFF-CBT concepts include psychoeducation, lowering expressed emotion in the household, and teaching cognitive-behavioral strategies to parents and children. Mnemonic "RAINBOW" is used to help participants remember the skills taught during treatment sessions. 
CFF-CBT requires direct involvement of parents/caregivers and would be contraindicated when this involvement cannot be assured.

\section{Family-Focused Therapy}

Family-focused treatment adapted for youth at high risk for bipolar disorder (FFT-HR) has shown promising results in children $\geq 9$ years of age. FFT-HR consists of 12 sessions over 4 months, with the possibility of booster sessions after acute treatment. Parents and children are involved together in therapy sessions. Sessions are grouped into three focus areas, which include psychoeducation (sessions 1-4), communication enhancement training (sessions 5-8), and problem-solving skills training (sessions 9-12). The communication section focuses on the negative effects highly expressed emotion has on patients with mood disorders.

In a development trial, 13 children (aged 9-17 years) with at least one parent with bipolar I or II disorder participated in FFT-HR along with their parents/caregivers [45]. The children met criteria for either major depression, cyclothymia, or bipolar disorder not otherwise specified. The primary outcome measure was the Adolescent Longitudinal Interval Follow-up Evaluation (ALIFE), which includes psychiatric status ratings (PSRs) on a 0-6 scale, which focuses on severity of mood symptom [46]. The youth involved in the study showed significant improvements in scores on the following sections of the ALIFE measure: depression severity, hypomania severity, and psychosocial functioning. A randomized controlled study examined the efficacy of FFT-HR in 40 youth, aged 9-17 years, with bipolar disorder not otherwise specified, MDD, or cyclothymia with active mood symptoms (YMRS score $>11$ or Children's Depression Rating Scale-Revised [CDRS-R] score $>29$ ) and at least one firstdegree relative with bipolar disorder type I or type II [47•,48]. The FFT-HR group was compared to an education control group (EC), which included one to two family sessions. Study participants received concomitant pharmacotherapy as necessary. Youth in the FFT-HR group responded more favorably than those in the EC group, with more rapid recovery and more weeks in remission as determined by the A-LIFE PRS ratings.

FFT-HR has been studied only in children who are at high risk for bipolar disorder (with at least one first-degree relative with bipolar disorder). It focuses on psychoeducation, communication skills, and problem-solving skills. It has not been studied in the preschool population, and findings cannot be generalized to very young children.

Treatment Duration

Treatment duration includes 12 sessions over the course of 4 months.

FFT requires direct involvement of parents/caregivers and would be contraindicated when this involvement cannot be assured. 


\section{Multifamily Psychoeducation}

Multifamily psychoeducation psychotherapy (MF-PEP) was developed by Fristad and colleagues as an adjunctive treatment for children with bipolar disorder [49]. Therapy is provided in groups that meet for eight sessions that are 90 min each in length. The focus of this therapy is to provide psychoeducation, lower expressed emotion within the family, teach cognitive-behavioral strategies to the children, and increase parent/caregiver concordance. Sessions begin and end with the families together, with the middle section including a separate parent/caregiver group and child group. Workbooks are provided to both parents/caregivers and children. Parent/caregiver groups include psychoeducation on mood disorders, their treatments, and the mental health care system, as well as teaching problem-solving skills, communication skills, and symptom management. The child groups consist of a similar psychoeducation component, focusing more on the disorder and treatment, and then teach cognitive-behavioral techniques, impulse-control training, and problem-solving and communication skills. MF-PEP has not yet been studied in preschool-aged children.

A randomized controlled trial examined the efficacy of MF-PEP in 165 children, aged 8-12 years, who had a diagnosis of major depression, dysthymia, or bipolar disorder type I, type II, or not otherwise specified [50••]. Of the 165 children, 78 received MF-PEP plus treatment as usual, and 87 received treatment as usual and served as the wait list control (WLC) group. The WLC group received treatment with MF-PEP at 12 months, after the immediate treatment group completed their treatment. The primary efficacy measure was the Mood Severity Index (MSI), calculated from the CDRS-R and the Mania Rating Scale (MRS) [48, 17]. The immediate treatment group showed significant decreases in MSI scores after completion of treatment compared to the WLC group. The WLC group showed similar decreases in their MSI scores a year later.

MF-PEP is a treatment provided to groups of families and patients which focuses on psychoeducation, lowering expressed emotion, fostering parental concordance, and providing cognitive-behavioral therapy to patients. There are two groups, a parent group and a patient group, and each group receives psychoeducation on diagnosis and treatment along with training in problem-solving and communication skills. Parents receive additional psychoeducation on the mental health system and symptom management, and children receive cognitive-behavioral therapy and impulse-control training. It has not yet been studied in preschool-aged children; therefore, results from the above studies cannot be generalized to this population.

Therapy consists of eight treatment sessions that are 90 min each; Therapy begins and ends with parents and children together, with the middle section consisting of separation of the two groups. 
MF-PEP requires direct involvement of parents/caregivers and would be contraindicated when this involvement cannot be assured.

Caution should be used when applying the above findings to very young children with bipolar disorder, as none of the studies include preschoolaged children (youngest age involved was 5 years old in the CFF-CBT studies). Also, these therapies are provided primarily as adjunctive treatment to medication. This is important, as the AACAP recommends treatment with psychotherapy before considering medications in the preschool age population $[31 \bullet \bullet]$. As such, there is a definite need for psychosocial treatment studies within this very young patient population to help better guide clinicians' treatment decisions.

\section{Pharmacological Interventions}

If psychotherapy is not beneficial in improving symptoms in preschoolaged children with bipolar disorder, and if symptoms are considered severe and impairing, then pharmacotherapy may need to be considered. A risk-benefit analysis is important before considering any medication, as side effects may be more of a concern in this young population. A meta-analysis of pharmacotherapy trials in pediatric patients with bipolar disorder showed overall modest effects of the various treatments (traditional mood stabilizers, other anticonvulsants, second-generation antipsychotics, and naturopathic compounds), although there was a lack of studies in children younger than 10 years. [51]. The few studies available including preschool-aged children are mostly open trials and will be reviewed below.

\section{Atypical Antipsychotics}

Most of the available studies in the preschool-aged population focus on atypical antipsychotics as a potential pharmacological intervention for bipolar disorder. In an open-label study examining the efficacy of aripiprazole in the treatment of bipolar disorder in very young children, 96 children, aged 4-9 years, with bipolar type I, type II, not otherwise specified, or cyclothymia were treated with aripiprazole for 16 weeks or until they met criteria for response [52]. Medication dosing was flexible (maximum daily dose was $15 \mathrm{mg} / \mathrm{mean}$ daily dose was $6.5 \mathrm{mg} /$ day), and there was significant symptom reduction in the participants over the course of the study, using the YMRS, CDRS-R, Clinical Global Impressions Scale-Severity (CGI-S) [53], and CGAS as the outcome measures. Out of the 96 study participants, $60(62.5 \%)$ completed the study and were considered a priori treatment responders (three of four consecutive weeks with CDRS-R $<29$, YMRS $<10$, and Children's Global Assessment Scale [CGAS] >50) [54]. At the end of the study, participants moved to the maintenance phase of the study, which was a double-blind, randomized, placebo-controlled study. These 60 children were randomized to receive either placebo or continued treatment with aripiprazole [55]. There was no significant difference in YMRS, CDRS-R, CGAS, or CGI-S scores between the two groups, but time to study discontinuation for 
any reason was significantly higher in the placebo group (3 mean weeks) than in the aripiprazole group (25.93 mean weeks). The rates of withdrawal from the study were high in both groups over the initial 4 weeks of the maintenance phase of the study, which suggested a nocebo effect or withdrawal due to the knowledge of possible switching from active treatment to placebo and the risk of relapse associated with this. The most commonly observed side effects in the aripiprazole group were stomach pain, increased appetite, and headaches. Mean weight gain in the aripiprazole group was $2.61 \mathrm{~kg}$.

An open-label examined the efficacy of quetiapine in 30 very young children (aged 4-6 years) with a diagnosis of bipolar type I, type II, or not otherwise specified with active mood symptoms (manic, hypomanic, or mixed) [56]. The mean endpoint dose of quetiapine was $175.8 \mathrm{mg} /$ day, and significant improvement in symptoms was seen over the course of the 8-week study, as determined by improvement in mean YMRS scores. Adverse effects included cold symptoms, sedation, GI upset, headache, decreased energy, and appetite increase, with mean weight gain of 3.1 lbs.

An open-label comparison trial examined the efficacy of olanzapine versus risperidone for the treatment of bipolar disorder in preschool-aged children [57]. This was an 8-week trial, including 31 children aged 4-6 years with a diagnosis of bipolar disorder type I, type II, or not otherwise specified. Both medications were effective at symptom reduction, as determined by significant reductions in YMRS scores with no difference between the two medications. Sixty-nine percent of children met response criteria (CGI-I score of "much" or "very much" improved or YMRS change of $\geq 30 \%$ ) in the risperidone group and $53 \%$ met response criteria in the olanzapine group. There was no significant difference in response rates between the two groups. Both groups experienced statistically significant weight gain, with no difference between the two groups. Commonly reported adverse effects in the study included appetite increase, common cold symptoms, headaches, and sedation.

There is a lack of studies examining the efficacy of traditional mood stabilizers in the treatment of preschool-onset bipolar disorder. The Treatment of Early Age Mania (TEAM) study was a randomized controlled trial that compared risperidone, lithium, and divalproex sodium for the acute treatment of bipolar disorder, manic or mixed, in 279 children aged 6 to 15 years [58••]. Risperidone was shown to be more effective than lithium or divalproex sodium, as measured by the Clinical Global Impressions for Bipolar Illness Improvement-Mania (CGI-BP-M) [41]. Response rates in the risperidone treatment group were $68.5 \%$ compared to 35.6 and $24 \%$ in the lithium and divalproex sodium groups, respectively, which was statistically significant. Metabolic side effects were a concern in the risperidone group. An open-label combination treatment study examined the efficacy of augmenting lithium treatment with risperidone in 38 children aged 4-17 with onset of bipolar disorder in preschool years [59]. There were 21 participants who were considered lithium non-responders after 8 weeks of monotherapy who received risperidone augmentation for up to 11 months. The remaining 17 children continued treatment with lithium monotherapy. The primary outcome measure was the YMRS, with response being defined as $\geq 50 \%$ decrease from baseline. The response rate was $86 \%$ in the combination treatment group. The 
most common adverse effects reported in both groups included weight gain, nausea/vomiting, appetite increase, stomach pain, and sedation.

\section{Contraindications}

Atypical antipsychotics are contraindicated in individuals with documented hypersensitivity to the drugs. Caution should be used when using certain atypical antipsychotics (risperidone, quetiapine) in patients with hepatic or renal impairment [35]. Ziprasidone is contraindicated in patients with familial long QT syndrome or history of other significant cardiovascular illnesses, and it should not be prescribed in combination with other agents known to prolong the QTc interval.

Drug Interactions

Carbamazepine may decrease the plasma concentration of certain atypical antipsychotics (risperidone, olanzapine, ziprasidone). Risperidone may increase plasma concentration of valproate. Fluoxetine and paroxetine may increase some atypical antipsychotic's plasma concentration (risperidone, aripiprazole).

Side Effects

Special Points

Children may be more vulnerable to the metabolic side effects of atypical antipsychotics, including potentially more significant weight gain in children versus adults treated with atypical antipsychotics [60•]. Other side effects include sedation, extrapyramidal side effects (EPS), neuroleptic malignant syndrome, tardive dyskinesia, hyperprolactinemia (more commonly with risperidone, olanzapine), and QTc prolongation (more commonly with ziprasidone). Quetiapine has shown risk of cataract development in animal studies. Clozapine carries the risk of agranulocytosis, seizures, and myocarditis. Monitoring guidelines exist to help guide clinicians in observing for metabolic risk factors and extrapyramidal symptoms [60•]. Correll and Carlson's healthy lifestyle guidelines are recommended to help reduce the risk of metabolic side effects in pediatric patients [61].

Medications should be considered after psychotherapeutic interventions have been attempted in the preschool population, and no medication has FDA approval for the treatment of bipolar disorder in children younger than age 10. Risperidone and aripiprazole are FDA approved for use in children aged 10-17 years for treatment of bipolar I disorder, manic or mixed. Olanzapine is FDA approved for use in adolescents aged 1317 years with bipolar I disorder, manic or mixed. Quetiapine is approved for use in mania associated with bipolar I disorder in children aged 1017 years. Ziprasidone and clozapine are not FDA approved for the treatment of bipolar disorder in children or adolescents. In preschool-aged children, dosing of atypical antipsychotics should begin at half the recommended dosage for children. Risperidone and aripiprazole are available in 
orally disintegrating tablet form and as oral solutions. Olanzapine is available in orally disintegrating tablet form.

Cost

Most of the above atypical antipsychotics are available in generic form, but they are still considerably expensive, especially for patients who do not have medication coverage through their insurance.

\section{Alternative Treatments}

A randomized controlled trial examined the efficacy of omega- 3 in the treatment of major depressive disorder in children aged 6-12 years [62]. In this 16week trial, omega-3 was significantly superior to placebo, with response rates ( $>50 \%$ reduction in CDRS-R) of $70 \%$ in the omega- 3 group versus $0 \%$ in the placebo group and remission rates (CDRSR $<29$ ) of $40 \%$ in the omega-3 group versus $0 \%$ in the placebo group. There were no clinically relevant side effects reported in the study. A 16-week randomized controlled trial of 51 pediatric patients aged 6-17 years with bipolar disorder type I or II examined the efficacy of flax oil (omega- 3 fatty acid, $\alpha$-linolenic acid) versus olive oil placebo adjunctively or as monotherapy in the treatment of mood symptoms [63]. There was no significant difference between the flax oil and placebo groups on outcome measures (change in YMRS, CDRS-R, and CGI ratings). Though there have been mixed results from the omega-3 fatty acid studies, the generable tolerability of this treatment in young school-aged children makes it a potential alternative treatment to consider in preschool-aged children with mood disorders. More studies examining its efficacy in this population are needed.

\section{Compliance with Ethics Guidelines}

\section{Conflict of Interest}

Melissa DeFilippis declares that she has no conflict of interest.

Dr. Wagner declares a non-compensated consultancy for Lundbeck. Dr. Wagner has also received honoraria not related to this article from UBM Medica, American Psychiatric Association, Las Vegas Psychiatric Society, American Academy of Child and Adolescent Psychiatry, NAC CME, Oxford University Press, Partners Healthcare, and Doctors Hospital at Renaissance and Slack, Inc.

\section{Human and Animal Rights and Informed Consent}

This article includes studies with human subjects performed by the authors. All studies had Institutional Review Board approval, and Informed Consent was obtained from all participants.

Wagner KD et al. A double-blind, randomized, placebo-controlled trial of escitalopram in the treatment of pediatric depression. J Am Acad Child Adolesc Psychiatry. 2006;45(3):280-288; Wagner KD et al. Efficacy of sertraline in the treatment of children and adolescents with major depressive disorder: two randomized controlled trials. Sertraline Pediatric Depression Study Group. JAMA. 2003;290(8):1033-1041; Wagner KD et al. A randomized, placebo-controlled trial of citalopram for the treatment of major depression in children and adolescents. Am J Psychiatry. 2004;161(6):1079-1083; Emslie GJ, Heiligenstein JH, Wagner KD, Hoog SL, Ernest DE, Brown E, Nilsson M, Jacobson JG. Fluoxetine for acute treatment of depression in children and adolescents: a placebo-controlled, randomized clinical trial. J Am Acad Child Adolesc Psychiatry. 2002;41(10):1205-1215) 


\section{References and Recommended Reading}

Papers of particular interest, published recently, have been highlighted as:

- Of importance

$\bullet \quad$ Of major importance

1. Fuhrman P, Equit M, Schmidt K, Gontard A. Prevalence of depressive symptoms and associated developmental disorders in preschool children: a population based study. Eur Child Adolesc Psychiatry. 2014;23:219-24.

2. $\quad$ Lavigne JV, Lebailly SA, Hopkins J, et al. The prevalence of ADHD, ODD, depression, and anxiety in a community sample of 4-year-olds. J Clin Child Adolesc Psychol. 2009;38(3):315-28.

This study was a very large community-based sample examining the incidence/prevalence of various disorders, including depression, among preschool-aged children. (Class III).

3. Dilsaver SC, Akiskal HS. Preschool-onset mania: incidence, phenomenology and family history. J Affect Disord. 2004;82S:S35-43.

4. Luby J, Belden A. Defining and validating bipolar disorder in the preschool period. Dev Psychopathol. 2006;18:971-88.

5. Hopkins J, Lavigne JV, Gouse KR, et al. Multi-domain models of risk factors for depression and anxiety symptoms in preschoolers: evidence for common and specific factors. J Abnorm Child Psychol. 2013;41:70522.

6. Egger HL, Erkanli A, Keeler G, et al. Test-restest reliability of the Preschool Age Psychiatric Assessment (PAPA). J Am Acad Child Adolesc Psychiatry. 2006;45:538-49.

7. Dougherty LR, Smith VC, Bufferd SJ, et al. Preschool irritability: longitudinal associations with psychiatric disorders at age 6 and parental psychopathology. J Am Acad Child Adolesc Psychiatry. 2013;52(12):1204313.

8. Dougherty LR, Bufferd SJ, Carlson GA, et al. Preschooler's observed temperament and psychiatric disorders assessed with a parent diagnostic interview. J Clin Child Adolesc Psychol. 2011;40(2):295-306.

9.• Luby JL, Gaffrey MS, Tillman R, et al. Trajectories of preschool disorders to full DSM depression at school age and early adolescence: continuity of preschool depression. Am J Psychiatry. 2014;171(7):768-76.

This is a large study that followed children with depressive symptoms from preschool- to school-age, with school-age depression occurring more commonly among the children with preschool-onset symptoms. It highlights the diagnostic continuity of depression among very young children. (Class III).

10. Angold A, Costello EJ. A test-retest reliability study of child-reported psychiatric symptoms and diagnoses using the Child and Adolescent Psychiatric Assessment (CAPA-C). Psychol Med. 1995;25:755-62.

11. Angold A, Colstello EJ. The Child and Adolescent Psychiatric Assessment (CAPA). J Am Acad Child Adolesc Psychiatry. 2000;39:39-48.

12. Luby JL, Belden AC, Pautsch J, et al. The clinical significance of preschool depression: impairment in functioning and clinical markers of the disorder. J Affect Disord. 2009;112(1-3):111-9.

13. Luby JL, Heffelfinger AK, Mrakotsky C, et al. Preschool major depressive disorder: preliminary validation for developmentally modified DSM-IV criteria. J Am Acad Child Adolesc Psychiatry. 2002;41(8):928-37.

14. Luby JL, Mrakotsky C, Heffelfinger A, et al. Modification of DSM-IV criteria for depressed preschool children. Am J Psychiatry. 2003;160:1169-72.

15. Gaffrey MS, Belden AC, Luby JL. The 2-week duration criterion and severity and course of early childhood depression: implications for nosology. J Affect Disord. 2011;133(3):537-45.

16. Demeter CA, Youngstrom EA, Carlson GA, et al. Age differences in the phenomenology of pediatric bipolar disorder. J Affect Disord. 2013;147:295-303.

17. Young RC, Biggs JT, Ziegler VE, Meyer DA. A rating scale for mania: reliability, validity, and sensitivity. Br J Psychiatry. 1978;133:429-35.

18. Youngstrom EA, Danielson CK, Findling RL, et al. Factor structure of the young mania rating scale for use with youths ages 5 to 17 years. J Clin Child Adolesc Psychol. 2002;31:567-72.

19. Luby JL, Belden AC. Clinical characteristics of bipolar vs. unipolar depression in preschool children: an empirical investigation. J Clin Psychiatry.

2008;69(12):1960-9.

20. Geller B, Tillman R, Bolhofner K. Proposed definitions of bipolar I disorder episodes and daily rapid cycling phenomena in preschoolers, school-aged children, adolescents, and adults. J Child Adolesc

Psychopharmacol. 2007;17(2):217-22.

21. Tillman R, Geller B. Definitions of rapid, ultrarapid, and ultradian cycling and of episode duration in pediatric and adult bipolar disorders: a proposal to distinguish episodes from cycles. J Child Adolesc

Psychopharmacol. 2003;13(3):267-71.

22. Eyberg SM, Funderburk BW, Hembree-Kigin TL, et al. Parent-child interaction therapy with behavior problem children: one and two year maintenance of treatment in the family. Child Fam Behav Ther. 2001;23:120.

23. Luby JL. Early childhood depression. Am J Psychiatry. 2009;166(9):974-9.

24. Lenze SN, Pautsch J, Luby JL. Parent-child interaction therapy emotion development: a novel treatment for depression in preschool children. Depression Anxiety. 2011;28:153-9.

$25 . \bullet \quad$ Luby JL, Lenze S, Tillman R. A novel early intervention for preschool depression: findings from a pilot 
randomized controlled trial. J Child Psychol Psychiatry. 2012;53(3):313-22.

This is a pilot randomized controlled trial examining the efficacy of a novel psychotherapeutic intervention for preschool depression, adapted from PCIT to address emotional regulation and competency. It shows this as a promising treatment for very young children with depression. (Class I).

26. Luby JL, Heffelfinger A, Koenig-McNaught AL, et al. The Preschool Feelings Checklist: a brief and sensitive screening measure for depression in young children. J Am Acad Child Adolesc Psychiatry. 2004;43:708-17.

27. Weisz JR, Southam-Gerow MA, Gordis EB, et al. Cognitive-behavioral therapy versus usual clinical care for youth depression: an initial test of transportability to community clinics and clinicians. J Consult Clin Psychol. 2009;77(3):383-96.

28. Kovacs M. Children's Depression Inventory (CDI): technical manual update. North Tonawanda: MultiHealth Systems; 2003.

29. Achenbach TM. Manual for the child behavior checklists/4-18 and 1991 profile. Burlington: University of Vermont; 1991.

30. Weisz JR, Thurber CA, Sweeney L, et al. Brief treatment of mild-to-moderate child depression using primary and secondary control enhancement training. J Consult Clin Psychol. 1997;65(4):703-7.

31.• Gleason MM, Egger HL, Emslie GJ, et al. Psychopharmacological treatment for very young children: contexts and guidelines. J Am Acad Child Adolesc Psychiatry. 2007;46(12):1532-72.

This reference includes the treatment guidelines put forth by the AACAP regarding treatment of very young children, including importance of utilizing psychotherapeutic interventions first, when possible. (Class IV).

32. Whittington CJ, Kendall T, Fonagy P, et al. Selective serotonin reuptake inhibitors in childhood depression: a systematic review of published and non-published data. Lancet. 2004;363:1341-5.

33. Hetrick S, Merry S, McKenzie J, et al. Selective serotonin reuptake inhibitors (SSRIs) for depressive disorders in children and adolescents. Cochrane Database Syst Rev. 2007;3, CD004851.

34. McClellan J, Werry J. Practice parameter for the assessment and treatment of children and adolescents with bipolar disorder. J Am Acad Child Adolesc Psychiatry. 2007;46(1):107-25.

35. Green WH. Child and adolescent clinical psychopharmacology. 4th ed. Philadelphia: Lippincott Williams \& Wilkins; 2007.

36. Emslie GJ, Heiligenstein JH, Wagner KD, et al. Fluoxetine for acute treatment of depression in children and adolescents: a placebo-controlled, randomized clinical trial. J Am Acad Child Adolesc Psychiatry. 2002;41(10):1205-15.

37. Wagner KD, Robb AS, Findling RL, et al. A doubleblind, randomized, placebo-controlled trial of escitalopram in the treatment of pediatric depression. J Am Acad Child Adolesc Psychiatry. 2006;45(3):280-8.
38. Wagner KD, Ambrosini P, Rynn M, et al. Efficacy of sertraline in the treatment of children and adolescents with major depressive disorder: two randomized controlled trials. Sertraline Pediatric Depression Study Group. JAMA. 2003;290(8):1033-41.

39. Wagner KD, Jonas J, Findling RL, et al. A randomized, placebo-controlled trial of citalopram for the treatment of major depression in children and adolescents. Am J Psychiatry. 2004;161(6):1079-83.

40. Pavuluri MN, Graczyk PA, Henry DB, et al. Child- and family-focused cognitive-behavioral therapy for pediatric bipolar disorder: development and preliminary results. J Am Acad Child Adolesc Psychiatry. 2004;43(5):528-37.

This is an open study showing the efficacy of CFF-CBT in a small sample of children and their families. This study included young children, aged 5 years and up, which is significant when considering treatments for the preschool-age population. (Class IV).

41. Spearing MK, Post RM, Leverich GS, et al. Modification of the Clinical Global Impressions (CGI) Scale for use in bipolar illness (BP): the CGI-BP. Psychiatry Res. 1997;73:159-71.

42. West AE, Henry DB, Pavuluri MN. Maintenance model of integrated psychosocial treatment in pediatric bipolar disorder: a pilot feasibility study. J Am Acad Child Adolesc Psychiatry. 2007;46(2):205-12.

This is a pilot study examining the feasibility of CFF-CBT in children with bipolar disorder and their caregivers. It is important because it includes children as young as 5 years old, the youngest age group included thus far in psychosocial therapies for pediatric bipolar disorder. (Class IV).

43. West AE, Jacobs RH, Westerholm R, et al. Child and family-focused cognitive-behavioral therapy for pediatric bipolar disorder: pilot study of group treatment format. J Can Acad Child Adolesc Psychiatry. 2009;18(3):239-46.

44. West AE, Weinstein SM, Peters AT, et al. Child-and family-focused cognitive-behavioral therapy for pediatric bipolar disorder: a randomized clinical trial. J Am Acad Child Adolesc Psychiatry. 2014;53(11):1168-78.

45. Miklowitz DJ, Chang KD, Taylor DO, et al. Early psychosocial intervention for youth at risk for bipolar I or II disorder: a one-year treatment development trial. Bipolar Disord. 2011;13(1):67-75.

46. Keller MB, Lavori PW, Friedman B, et al. The longitudinal interval follow-up evaluation: a comprehensive method for assessing outcome in prospective longitudinal studies. Arch Gen Psychiatry. 1987;44:540-8.

47. Miklowitz DJ, Schneck CD, Singh MK, et al. Early intervention for symptomatic youth at risk for bipolar disorder: a randomized trial of family-focused therapy. J Am Acad Child Adolesc Psychiatry. 2013;52(2):12131.

This is a randomized controlled trial examining the efficacy of FFT-HR in children at risk for bipolar disorder. Though it does not include children younger than age 9, it is still an important treatment to consider, even in younger children with bipolar disorder. (Class I). 
48. Poznanski EO, Grossman JA, Buchsbaum Y, et al. Preliminary studies of the reliability and validity of the Children's Depression Rating Scale. J Am Acad Child Psychiatry. 1984;23(2):191-7.

49. Fristad MA, Gavazzi SM, Mackinaw-Koons B. Family psychoeducation: an adjunctive intervention for children with bipolar disorder. Biol Psychiatry. 2003;53:1000-8.

$50 . \bullet$ Fristad MA, Verducci JS, Walters K, Young ME. Impact of multifamily psychoeducational psychotherapy in treating children aged 8 to 12 years with mood disorders. Arch Gen Psychiatry. 2009;66(9):1013-21.

This is a randomized controlled trial with a large sample size showing efficacy of MF-PEP as an adjunctive treatment for bipolar disorder in school-aged children. (Class I).

51. Liu HY, Potter MP, Woodworth KY, et al. Pharmacologic treatments for pediatric bipolar disorder: a review and meta-analysis. J Am Acad Child Adolesc Psychiatry. 2011;50(8):749-62.e39.

52. Findling RL, McNamara NK, Youngstrom EA, et al. An open-label study of aripiprazole in children with a bipolar disorder. J Child Adolesc Psychopharmacol. 2011;21(4):345-51.

53. National Institute of Mental Health. Clinical Global Impressions Scale. Psychopharmacol Bull. 1985;21:839-43.

54. Shaffer D, Gould MS, Brasic J, et al. A Children's Global Assessment Scale (CGAS). Arch Gen Psychiatry. 1983;40:1228-31.

55. Findling RL, Youngstrom EA, McNamara NK, et al. Double-blind, randomized, placebo-controlled longterm maintenance study of aripiprazole in children with bipolar disorder. J Clin Psychiatry. 2012;73(1):57-63.

56. Joshi G, Petty C, Wozniak J, et al. A prospective openlabel trial of quetiapine monotherapy in preschool and school age children with bipolar spectrum disorder. J Affect Disord. 2012;136:1143-53.

57. Biederman J, Mick E, Hammerness P, et al. Open-label, 8 -week trial of olanzapine and risperidone for the treatment of bipolar disorder in preschool-age children. Biol Psychiatry. 2005;58:589-94.

58.• Geller B, Luby JL, Joshi P, et al. A randomized controlled trial of risperidone, lithium, or divalproex sodium for initial treatment of bipolar I disorder, manic or mixed phase, in children and adolescents. Arch Gen Psychiatry. 2012;69(5):51528.

This is a randomized controlled trial including children as young as 6 years old that shows the superiority of risperidone to traditional mood stabilizers lithium and divalproex sodium. It also highlights the concern of metabolic effects in such a young age group when treated with atypical antipsychotics. (Class I).

59. Pavuluri MN, Henry DB, Carbray JA, et al. A one-year open-label trial of risperidone augmentation in lithium nonresponder youth with preschool-onset bipolar disorder. J Child Adolesc Psychopharmacol. 2006;16(3):336-50.

60. Correll CU. Antipsychotic use in children and adolescents: minimizing adverse effects to maximize outcomes. J Am Acad Child Adolesc Psychiatry. 2008;47(1):9-20.

This article is important because it provides a thorough overview of the observed adverse effects with antipsychotic use in children and adolescents and provides guidelines for the clinician to follow with respect to monitoring and clinical work up that may be necessary to help prevent bad outcomes in this vulnerable population. (Class IV).

61. Correll CU, Carlson HE. Endocrine and metabolic adverse effects of psychotropic medications in children and adolescents. J Am Acad Child Adolesc Psychiatry. 2006;45(7):771-91.

62. Nemets H, Nemets B, Apter A, et al. Omega-3 treatment of childhood depression: a controlled, double-blind pilot study. Am J Psychiatry. 2006;163:1098-100.

63. Gracious BL, Chirieac MC, Costescu S, et al. Randomized, placebo-controlled trial of flax oil in pediatric bipolar disorder. Bipolar Disord. 2010;12(2):142-54. 